\title{
ANSIEDADE ENTRE DESEMPREGADOS COM FOCO EM DIFERENCIAIS DE GÊNERO
}

\author{
Priscilla Pinto Araujo ${ }^{1}$; Tânia Maria de Araújo $^{2}$; \\ 1. Bolsista PIBIC/CNPq, Graduanda em Psicologia, Universidade Estadual de Feira de Santana, e-mail: \\ priscilla_tuca@hotmail.com \\ 2. Orientadora, Departamento de Saúde, Universidade Estadual de Feira de Santana, e-mail: \\ araujo.tania@uefs.br
}

PALAVRAS-CHAVE: desemprego, gênero, ansiedade.

\section{INTRODUÇÃO}

A concepção de trabalho transcende a ideia de fonte de sustentação econômica e material; o trabalho expressa também, uma perspectiva ontológica e psicossocial, onde há a constituição de identidades, de representação subjetiva, realização pessoal e humanização do mundo e das atividades nele executadas (Moura, 1998; Antunes, 1999). Em virtude disso, torna-se relevante considerar os impactos que o trabalho, sob determinadas condições, pode ter sobre a saúde, assim como, a sua ausência (no caso, o desemprego) pode implicar negativamente na saúde mental dos indivíduos (Pinheiro; Monteiro, 2007).

O desemprego constitui um problema para além do indivíduo, sua gênese está associada a fatores macrossociais, históricos (Barros, Oliveira, 2009) e principalmente, envolvem dimensões de classe, institucionais, biográficas e subjetivas (Souza, 2010). A relação entre o desemprego e a saúde mental pode ser entendida pelo sentido positivo do trabalho para cada indivíduo, como, por exemplo, a troca de experiências e contato com outras pessoas. Em uma situação de desemprego esses aspectos, que são determinantes para a saúde mental, podem ser afetados ou até mesmo perdidos (Uhmp, 2000). Em consequência disso, entre os efeitos psicológicos do desemprego na saúde mental dos indivíduos, destaca-se a insatisfação com a vida, baixa autoestima, problemas familiares e, sobretudo, a ocorrência de transtornos mentais como a ansiedade (Warr 1987; Hammarstrom, 1994).

Tendo em vista que as investigações de estimativas de prevalência de transtornos mentais na população geral são de suma importância para a saúde pública, avaliar as implicações do desemprego na saúde mental de homens e mulheres, se faz necessário na medida em que a condição do indivíduo nas sociedades depende da sua inserção nos sistemas de trabalho. Quando esse sistema é rompido, resulta para quem o vivencia, na sensação de fracasso pessoal, desqualificação social, marginalização, insegurança, angústia, crises e tensões, favorecendo dessa forma, à ocorrência de ansiedade.

Considerando que a pesquisa "Caracterização da Situação de Saúde Mental em Feira de Santana, Bahia, Brasil" busca contribuir para um melhor dimensionamento dos agravos à saúde mental da população de Feira de Santana, e possibilitar a construção de políticas públicas para esse campo, este estudo teve como objetivo avaliar fatores associados a ansiedade entre homens e mulheres em situação de desemprego do município de Feira de Santana, Bahia.

\section{MATERIAL E MÉTODOS}

Este estudo é um recorte da pesquisa "Caracterização da Situação de Saúde Mental em Feira de Santana, Bahia, Brasil." Trata-se de um estudo epidemiológico do tipo corte transversal, realizado pelo Núcleo de Epidemiologia da Universidade Estadual de Feira de Santana, BA. 
A população deste estudo foi constituída de uma amostra selecionada, por procedimento aleatório, da população com idade de 15 anos ou mais, residente na zona urbana de Feira de Santana, Bahia, Brasil. A seleção das áreas de estudo foi feita por amostragem por conglomerados, estratificada por subdistritos mediante critérios aleatórios, com base em dados censitários da Fundação Instituto Brasileiro de Geografia e Estatística. Todos os indivíduos com 15 anos ou mais de idade foram considerados como população de estudo e convidados a participar do mesmo.

Para avaliação da saúde mental, foi utilizado o instrumento de entrevista clínica estruturada, o Patient Health Questionnaire (PHQ) para mensurar a ansiedade em homens e mulheres em situação de desemprego de Feira de Santana, Bahia. O PHQ é um instrumento derivado do estudo PRIME-MD (Primary Care Evaluation of Mental Disorders), e destina-se à detecção dos cinco tipos de transtornos mentais (depressão, ansiedade, humor, abuso de substâncias e alimentares) (Spitzer, Kroenke, Williams, 1999). É assim, um instrumento capaz de estabelecer diagnóstico específico desses cinco tipos de morbidade psíquica.

A variável de desfecho nesse estudo foi a ansiedade em homens e mulheres desempregados de Feira de Santana, Bahia, avaliada pelo PHQ. Essa variável foi composta por meio de sete itens do PHQ, dispostos em uma escala de três pontos: 0 "nenhuma vez", 1- "vários dias" e 2- "mais da metade dos dias". Os sete itens foram dicotomizados em sim e não. Para esta dicotomização foi empregado um ponto de corte para avaliação de ansiedade: quando o indivíduo respondeu sim para a pergunta 5. (a) ou mais de três respostas como "mais da metade dos dias" para os itens $5 \mathrm{~b}$ a $5 \mathrm{~g}$, sendo considerado um caso nesta situação. A definição de não caso foi feita quanto se respondeu não para a pergunta $5 \mathrm{a}$ ou menos de três respostas "mais da metade dos dias" para os itens de $5 \mathrm{~b}$ a $5 \mathrm{~g}$ (Spitzer et al., 2000).

A variável de exposição de interesse, desemprego, foi avaliada pela resposta às questões: "Você trabalha atualmente?" - Em caso negativo, indagou-se qual a situação atual? Dentre as opções estava a opção "estou desempregado". Considerou-se, neste estudo, como desempregado quem afirmou estar nessa condição.

Para a análise das variáveis foi utilizado o programa estatístico "Statical Package for the Social Science" (SPSS) for Windows, versão 9.0 e o EpiInfo versão 7.0.

Inicialmente a análise de dados compreendeu a caracterização da população estudada. Em seguida, foi calculada a prevalência global de ansiedade. A descrição foi feita para toda a população e separadamente para homens e mulheres. Após essa caracterização realizou-se análise bivariada.

$\mathrm{Na}$ análise de associação entre o desfecho e as variáveis de exposição, foram calculadas Razões de Prevalência (RP) e os respectivos Intervalos de Confiança de 95\%. Utilizou-se o teste Qui-Quadrado de Person $\left(X^{2}\right)$ para avaliação de significância estatística, adotando valor de $\mathrm{p} \leq 0,05$.

Os protocolos de pesquisa foram avaliados e aprovados pelo Comitê de Ética em Pesquisa com Seres Humanos da Universidade Estadual de Feira de Santana sob parecer $\mathrm{n}^{\circ} 042 / 06$. A apresentação dos resultados neste estudo assegura o anonimato dos notificados e investigados.

\section{RESULTADOS E/OU DISCUSSÃO}

A prevalência global de ansiedade foi de 16,6\%. Identificou-se maior prevalência de ansiedade entre as mulheres $(18,6 \%)$ em comparação aos homens $(10,5 \%)$.

$\mathrm{Na}$ avaliação dos sintomas ansiosos, as maiores prevalências estavam relacionadas aos itens "ter dificuldade para pegar no sono ou permanecer dormindo" $(28,6 \%)$, "sentir-se agitado/a sendo difícil ficar sentado/a" (30,7\%) e "ficar facilmente aborrecido/a ou irritado/a" (43,9\%). Esses resultados revelam que em situação de 
desemprego, os indivíduos passam a apresentar sentimentos de insatisfação com a vida, irritabilidade e distúrbios do sono, evidenciando elevados níveis de ansiedade.

Identificou-se maior prevalência de ansiedade entre as mulheres $(18,6 \%) \mathrm{em}$ comparação aos homens (10,5\%). Levando em conta o nível educacional, constatou-se elevada prevalência na condição ausência de escolaridade $(50,0 \%)$ entre os homens e nível fundamental $(28,4 \%)$ entre as mulheres. As diferenças foram estatisticamente significantes.

Em referência a situação de desemprego, a prevalência de ansiedade foi maior, em homens $(13,9 \%)$ e mulheres $(21,9 \%)$ que estavam desempregados há mais de 12 meses. As diferenças nas prevalências de acordo com o tempo de desemprego, não foram estatisticamente significantes. Contudo, apesar dos resultados não identificarem associações significantes, vale salientar que quanto maior for o tempo de desemprego, maiores são as implicações negativas na saúde mental do indivíduo.

Os valores de p revelaram diferenças estatisticamente significante na avaliação de ansiedade conforme hábitos de vida "participa de alguma atividade de lazer para se divertir ou distrair?" ( $\mathrm{P}<0,00)$ e estado de saúde "pressão alta" $(\mathrm{P}<0,00)$; "distúrbio do sono" $(\mathrm{P}<0,02)$ entre homens em situação de desemprego.

A presença de ansiedade em desempregados segundo diferenciais de gênero, implica negativamente na saúde e na qualidade de vida de homens e mulheres, considerando que essa situação recai na falta de confiança em si e nos outros, instabilidade emocional, visão pessimista da vida e sentimentos de ineficácia frente à sua condição (Burgard; Brand; House, 2007;)

Socialmente, o nível educacional reflete em um fator significativo ao processo de empregabilidade. A literatura aponta que o desemprego incide em grande parte, sobre homens e mulheres de baixa escolaridade e/ou sem escolarização (Bernardim, 2001). As transformações de mercado irão exigir gradativamente mais habilidades para o trabalho, e em razão disso, substitui-se grupos menos escolarizados por àqueles com maior escolarização (Saviani, 1994). Diante desse momento de crise, emerge uma situação preocupante que é vista como ameaçadora pelo indivíduo, e por esse motivo, acaba desencadeando reações de ansiedade. Consequentemente, em função das determinações sociais de papéis de masculinidade, os homens, particularmente, expressam sentimentos depreciativos sobre si próprio, como a vergonha, impotência e/ou ineficácia (Borcsik, 2006).

A ausência de emprego afeta diretamente o orçamento financeiro, o que torna necessário ao indivíduo o ajustamento e a modificação de modelos e estilos de vida anteriores à situação de desemprego. Contudo, nesse processo de mudança, nota-se a frustação e insatisfação do sujeito em virtude da precariedade do lazer e da redução da sua qualidade de vida (Aguiar et al, 2017). Dessa maneira, percebe-se que atrelado a privação material, a falta de autorrealizaçao e as limitações sociais, a ausência de atividades de lazer em função do desemprego, pode ocasionar ansiedade, e esta, por sua vez, compromete a saúde dos homens, interferindo na execução de inúmeras atribuições diárias e produzindo uma série de questões biopsicossociais (Araújo, et al., 2007).

$\mathrm{O}$ indivíduo quando está ansioso apresenta um conjunto de reações fisiológicas referentes a uma situação ameaçadora. De acordo com Quadros et al., (2006), enquanto o organismo prepara as respostas comportamentais frente a situações de ameaça ou perigo, o corpo experimenta sintomas físicos relacionados ao aumento da frequência cardíaca, do consumo de oxigênio, da pressão arterial e da frequência respiratória. Atentando-se a essa perspectiva, sugere-se que, em comparação as mulheres, os homens possuem níveis mais elevados de ansiedade, tendo em vista que na gravidade dos sintomas ansiosos o sujeito sente palpitações, tonturas, pressão alta e problemas com o sono (Mckenzie, 2015). 


\section{CONSIDERAÇÕES FINAIS}

A avaliação da saúde mental de homens e mulheres desempregados, possibilitou um conhecimento ampliado a respeito dos fatores associados a essa situação, assim como, do impacto socioeconômico e psicológico nos modos de vida e saúde na população investigada.

Esse estudo constatou estimativas elevadas de ansiedade entre desempregados no município de Feira de Santana, Bahia. Em consequência disso, o conhecimento da prevalência de ansiedade nessa população, permite pensar possibilidades e/ou limites de atuação das políticas públicas no desenvolvimento de estratégias de enfrentamento diante de uma situação de desemprego, para que assim, seja possível proporcionar a homens e mulheres condições e recursos estruturais, materiais e psicossociais, com o intuito de ajudá-los a lidar com essa circunstância estressora, além de promover melhor qualidade de vida à população.

\section{REFERÊNCIAS}

AGUIAR, J.; et al (2017) Avaliação da Experiência de Estar em Desemprego: Pontos Positivos e Negativos. Cad. Ed. Tec. Soc., v.10, n.1, p. 28-39.

ARAÚJO, T. M.; et al (2007). Prática de Atividades de Lazer e Morbidade Psíquica em Residentes de Áreas Urbanas. Revista Baiana de Saúde Pública, v.31, n.2, p.294-310.

BARROS, C. A; OLIVEIRA, T. L (1998). Saúde mental de trabalhadores desempregados. RPOT, v.9, n.1, p.86-107.

BERNARDIM,M. L (2001). A escolarização e suas relações com a condição de emprego e desemprego. Abbra-se a novas idéias, v. Ii, p. 47-62.

BORCSIK, Sonia Pacheco de Lima (2006). Avaliação da ansiedade e do enfrentamento de executivos em situação de desemprego. São Bernado do Campo, 2006. 63p. Dissertação (Mestrado em Psicologia) - Universidade Metodista de São Paulo.

BURGARD, S.A; BRAND, J.E.; HOUSE, J.S. (2007). Toward a better estimation of the effect of job loss on health. Journal of Health and Social Behavior, 48, 369-384.

MCKENZIE, Professor Dr. Kwame (2010). Ansiedade e Ataques de Pânico. São Paulo, 2010, São Paulo. Tese (Doutorado em Sociologia), São Paulo, USP, 2010.

MOURA, P. C (1998). A crise do emprego: uma visão para além da economia. Rio de Janeiro: Mauad.

PINHEIRO, Letícia Ribeiro Souto; MONTEIRO, Janine Kieling (2007). Refletindo sobre desemprego e agravos à saúde mental. Cad. psicol. soc. trab., São Paulo, v. 10, n. 2, p. 35-45.

QUADROS, A. C. J. et al (2006). Relações entre ansiedade e psicologia do esporte. Revista digital, Buenos Aires, Ano 11, n. 98.

SAVIANI, Dermeval (1994). O trabalho como princípio educativo frente às novas tecnologias. In: FERRETTI, Celso J. et al. Novas tecnologias, trabalho e educação: um debate multidisciplinar. Rio de Janeiro : Vozes.

SOUZA, D. C. C. de (2015). Sindicalismo e desempregados no Brasil e argentina de 1990 a 2002: unidade e fratura entre o exército de operários ativo e de reserva. SP: Editora Fundamento Educacional Ltda.

SPITZER R. L., KROENKE K., WILLIAMS J (1999). B. Validation and utility of a self-report version of PRIME-MD: The PHQ Primary Care Study. JAMA n.282, p.1737-44. 
UNEMPLOYMENT AND MENTAL HEALTH PROJECT. Framework for the project on unemployment and mental health. 2000. Disponível em <www.uhmp.org>. Acesso em: 15 ago. 2018.

WARR, Peter (1987). Work, unemployment and mental health. Oxford: Clarendon Press. 